Chapter 20

\title{
Astrocytes Role in Parkinson: A Double-Edged Sword
}

\author{
Ricardo Cabezas, Marco Fidel Avila, Daniel Torrente, \\ Ramon Santos El-Bachá, Ludis Morales, \\ Janneth Gonzalez and George E. Barreto
}

Additional information is available at the end of the chapter

http://dx.doi.org/10.5772/54305

\section{Introduction}

Parkinson Disease (PD) is the second most chronic neurodegenerative disorder in the world, after Alzheimer's Disease (AD), and is estimated to affect about $1 \%$ of the population over 60 years of age. PD is caused by the disruption of dopaminergic neurotransmission in the basal ganglia, which causes a reduction in the numbers of dopaminergic neurons in the substantia nigra and formation of cytoplasmic inclusions called Lewy bodies [1].

Both in normal and pathological circumstances, astrocytes are critical supporters of neuronal function in processes such as antioxidant protection, glutamate clearance, the development and/or maintenance of blood brain barrier characteristics, the release of gliotransmitters and cytokines [2-4]. In recent years, much research on PD has focused on the astrocytic-neuronal crosstalk, suggesting that this interaction is important for future therapies against neurodegenerative processes. During brain damage events, astrocytes become transiently or permanently impaired, and the subsequent impact on neuronal cells may lead to pathological conditions such as PD [5-7].

In the present chapter, we provide a brief overview of the astrocytic functions and the pathophysiological events elicited during PD. Additionally, we explore the beneficial and damaging consequences of reactive astrogliosis in dopaminergic neurons during PD, particularly on oxidative damage, which is a main component of numerous neuropathological conditions, and that may have a damaging effect in astrocytic functions. We also highlight some of the cellular and animal models currently used in Parkinson research, such rotenone, 1methyl-4-phenyl-1,2,3,6-tetrahydropyridine (MPTP) and paraquat as inducers, which have many similar features with this disease. Finally, a brief overview of the future perspectives in astrocytic protection during Parkinson development is discussed. 


\section{Parkinson's disease}

PD is a progressive neurodegenerative disorder caused by the neuronal death in the substantia nigra (SN), degeneration of dopaminergic neurotransmission, and the presence of $\alpha$ synuclein and protein inclusions in neuronal cell bodies (Lewy bodies) [4-5,7]. Main symptoms of Parkinson are asymmetrical bradikinesia, rigidity, resting tremor and postural instability. Other non-motor symptoms that generate serious disability problems have also been noted, including fatigue, pain, Lewy Body dementia, psychosis, depression, and apathy [1]. Although there is not a cure for the disease, the most used and cheaper treatment for PD continues to be Levodopa $[1,8]$. However, about $40 \%$ of patients developed motor fluctuations and dyskinesias after 4 to 6 years of treatment [1], demonstrating that further pharmacological research is needed in order to counterbalance side effects. In this aspect, treatments using long-acting dopaminergic agents or a continuous dopaminergic effect in the striatum have been associated with less severe motor complications, given alone or in combination with L-dopa [9]. Some pharmacological agents that have shown promising applications, include dopamine agonist like apomorphine and ropinirole, and catechol-Omethyltransferase (COMT; EC 2.1.1.6) inhibitors [9].

Numerous reviews and articles agree that the exact cause of PD remains unknown [1,9-10]. Mutations in various proteins such as leucine-rich repeat kinase 2 (LRRK2; EC 2.7.11.1), Parkinson protein 2 (PARK2), probable cation-transporting ATPase type 13A2 (ATP13A2; EC 3.6.3-), phosphatase and tensin homolog (PTEN)-induced putative kinase 1 (PINK1; EC 2.7.11.1), and Parkinson disease (autosomal recessive, early onset) 7 protein (DJ-1) have been observed in familiar cases of Parkinson, which only accounts for $10-15 \%$ of diagnosed cases [6,11-12]. Interestingly, LRRK2, PINK1, and DJ-1, which are present in mitochondrial membranes, have been suggested to play a role in reactive oxygen species (ROS) production by a defective maintenance of the mitochondrial membrane potential [12-13].

A number of environmental factors have been found to induce PD-like symptoms, and are currently used in animal and cellular models of the disease. Environmental factors include vascular insults to the brain, oxidative stress, neuroleptic drugs and repeated head trauma. $[6,14]$. Additionally, the exposure to pesticides like rotenone or 1-methyl-4-phenylpyridinium $\left(\mathrm{MPP}^{+}\right)$and heavy metals (manganese) increases the risk of PD development $[6,10$, 14-15]. In this aspect, numerous epidemiologic and toxicologic studies have examined pesticides as a risk factor for PD and parkinsonism and the possible mechanisms by which pesticides may act [14-17].

Initiation and progression of PD is dependent upon cellular events, including failures in the protein degradation machinery, oxidative stress, mitochondrial dysfunction, defects in mitochondrial autophagy (mitophagy) and the continuous accumulation of $\alpha$-synuclein, driven through cell to cell interactions between glial cells and neurons that ultimately lead to apoptosis $[7,10,18]$. Previous studies pointed that astrocytic $\alpha$-synuclein deposition initiates the recruitment of phagocyte microglia that attacks and kills neurons in restricted brain regions $[7,19]$, correlating this $\alpha$-synuclein accumulation with nigral neuronal cell death [20], and suggest the importance of astrocytes in the initiation of the disease. Conversely, astrocytes 
also have beneficial roles during PD progression [21-22]. For example, astrocytes express different antioxidant molecules such as glutathione peroxidase (EC 1.11.1.9), which have been inversely correlated with the severity of dopaminergic cell loss in the respective cell groups in patients with PD [4].

\section{Astrocytes in PD}

\subsection{Astrocytic functions}

Astrocytes are the most common cell type in the mammalian brain, conforming the glia with oligodendrocytes and microglia [23]. They are characterized by the expression of the intermediate filaments glial fibrillary acidic protein (GFAP) and vimentin (Vim). Astrocytes are essential for the metabolism of the brain, transporting multiple nutrients and metabolic precursors to the neurons by the malate-asparte shuttle and other transporters [24]. There are two main types of astrocytes in the SNC: Protoplasmic astrocytes, which envelope neuronal bodies and synapses and fibrous astrocytes which interact with the nodes of Ranvier and oligodendroglia [7]. Current research has shown that only protoplasmic astrocytes have an increase in the accumulation of $\alpha$-synuclein, whereas fibrous astrocytes do not $[7,19]$.

Current knowledge indicates that astrocytes are critical for some cellular processes, such as the development and/or maintenance of blood-brain barrier characteristics, the promotion of neurovascular coupling, the attraction of cells through the release of chemokines, $\mathrm{K}^{+}$buffering, release of gliotransmitters, release of glutamate by calcium signaling, maintenance of general metabolism, control of the brain $\mathrm{pH}$, metabolization of dopamine and other substrates by monoamine oxidases (MAOs; EC 1.4.3.4), uptake of glutamate and $\gamma$-aminobutyric acid (GABA) by specific transporters and production of antioxidants [2-3,25-27] (Figure 1). Recent evidence has shown that astrocytes are arranged in non-overlapping domains forming a syncytial network that may contact approximately 160.000 synapses, thus integrating neural activity with the vascular network [4,28]. In this aspect, astrocytic terminal processes, known as endfeet, contact the brain vasculature and enwrap the neuronal synapses, enabling the modulation of both neuronal activity and cerebral blood flow, following an elevation in intracellular $\mathrm{Ca}^{2+}$ levels in the endfeets [24,29].

During brain damage (including diseases, brain injury and oxidative stress), these astrocytic functions become transiently or permanently impaired, and the subsequent impact on neuronal cells may lead to pathological conditions and neurodegenerative diseases $[3,26]$. Neurons are more susceptible to injury than astrocytes, as they have limited antioxidant capacity, and rely heavily on their metabolic coupling with astrocytes to combat oxidative stress [3]. However, severe brain damage also results in astrocyte dysfunction, leading to increased neuronal death [30].

As previously stated, astrocytes exert both neuroprotective and neurodegenerative roles, depending on the molecules released by them, and the pathological or normal circumstances of their microenvironment [6]. For example, astrocytes release antioxidant molecules like 
glutathione (GSH) and superoxide dismutases (SODs; EC 1.15.1.1), and supply neurons with neurotrophic factors, such as nerve growth factor (NGF), basic fibroblast growth factor (bFG), that constitute an important attempt to protect neurons during brain damaging processes, including PD [6, 31-32]. On the other hand, during the process of reactive astrogliosis, astrocytes release inflammatory cytokines that may affect the surrounding neurons, both by the induced production of ROS and lipid peroxidation, and by the activation of apoptotic mechanisms that induce neuronal dopaminergic death $[6,10]$. These unusual, and sometimes contradictory, features of astrocytes in PD will be further explored in this chapter.

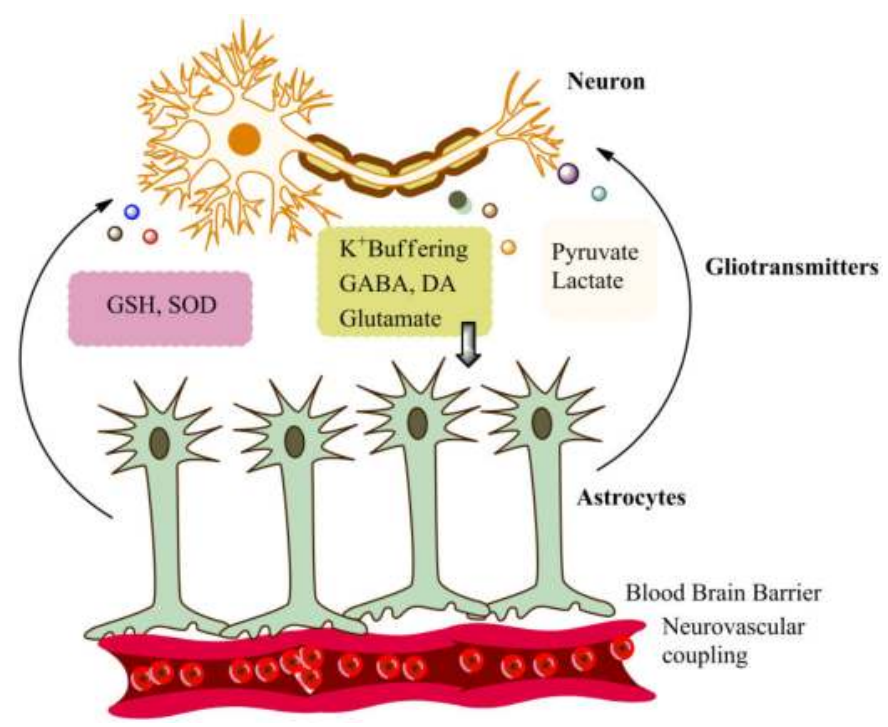

Figure 1. Astrocytes support neuronal function by multiple ways, including the development and maintenance of blood-brain barrier and promoting the neurovascular coupling. Astrocytes regulate the levels of ions, neurotransmitters and fueling molecules such as $\mathrm{K}^{+}$, glutamate, GABA, dopamine, lactate and piruvate. Furthermore, astrocytes promote the attraction of cells through the release of chemokines, and produce beneficial antioxidants, including glutathione, superoxide dismutases (SODs 1, 2 and 3), and ascorbate.

\subsection{Astrogliosis and parkinson}

Reactive astrogliosis is the main reaction of astrocytes following brain insults such as infection, trauma [33-34], $\alpha$-synuclein accumulation [35], ischemia [36-37] and neurodegenerative diseases [3]. This process involves both molecular and morphological changes in the astrocytes, including increased expression of GFAP, vimentin and nestin, uptake of excitotoxic glutamate, protection from oxidative stress by the production of GSH, neuroprotection by release of adenosine, degradation of amyloid-beta peptides, facilitation of blood-brain barrier, increased formation of gap junctions between astrocytes, formation of scars and, in some cases release of inflammatory cytokines, including tumor necrosis factor- $\alpha$ (TNF- $\alpha$ ), and production of ROS [3,35,38-40]. 
Astrogliosis and microgliosis in the SN of Parkinson patients are key features of the disease, which is a nonspecific consequence of neuronal degeneration [10]. Cellular and animal models using environmental and biological toxins, especially lipopolysaccharides (LPS), herbicides and pesticides like rotenone or MPTP (1-methyl-4-phenyl-1,2,3,6tetrahydropyridine), can induce both astrogliosis and microgliosis, which is accompanied by neuronal death, mitochondrial dysfunction and nuclear fragmentation [41-45]. Additionally, it was previously shown that the injection of LPS in rat brains was followed by an increase in the inducible nitric oxide synthase (iNOS; EC 1.14.13.39), suggesting that chronic glial activation can cause oxidative stress in the brain, similarly to that seen in neurodegenerative processes like AD and Parkinson [10, 39, 45]. A previous report showed that activated glial cells can participate in the death of dopaminergic neurons, probably by the activation of apoptosis by cytokines like TNF- $\alpha$, IL-1B, IL- 6 and interferon- $\gamma$ and the subsequent production of nitric oxide (NO) by the iNOS that may diffuse toward the neurons and induce lipid peroxidation, DNA strands breaks and inhibition of mitochondrial metabolism [6,10]. Furthermore, cytokines released by astrocytes may bind to their specific receptors in the dopaminergic neurons, such as TNFR1 and 2, and activate proapoptotic mechanisms through the activation of caspase 3, caspase 8, and cytochrome c [10]. Interestingly, the excessive uptake of neuronal $\alpha$-synuclein by astrocytes can lead to accumulation of aggregates of this protein in astrocytes, and cause an upregulation of IL- $1 \alpha$, IL- $1 \beta$ and IL- 6 , followed by the release of TNF- $\alpha$ and IL-6 [6]. These results suggest that the inhibition of glial reaction to damage and further inflammatory processes could be considered as a promising therapy to reduce neuronal damage during PD [10].

\subsection{Oxidative stress and Parkinson: Role of astrocytes}

In the brain, oxidative stress and other toxic insults can trigger the overexpression and activation of neuronal nitric oxide synthase that increases NO production and may cause apoptotic cell death by inducing the release of cytochrome $\mathrm{c}$ from mitochondrial impairment, loss of membrane potential, the opening of permeability transition pores, and the release of proapoptotic molecules [46,47]. After brain damaging processes, neurons experience greater metabolic deterioration than glial cells. For instance, astrocytes contain glycogen stores that allow them to maintain ATP production through glycolysis and mitochondrial membrane potential by reversal of the F0-F1-ATPase (EC 3.6.3.14) [48]. For example, cultured astrocytes subjected to oxygen and glucose deprivation showed a decrease in mitochondrial membrane potential, possibly caused by the mitochondrial permeability transition pore (mtPTP) opening, which leads to a loss of intramitochondrial contents, mitochondrial respiration and ATP production [48].

Nowadays there is much evidence of the role of oxidative stress in the development of neurodegenerative diseases, such as AD, PD, Amyotrophic Lateral Sclerosis (ALS) and Huntington's disease (HD). Much of these oxidative damaging processes are associated with an imbalance on the production of ROS that leads to mitochondrial stress and impairment in energy production $[47,49]$. ROS, such as superoxide $\left(\mathrm{O}^{\bullet} 2^{-}\right)$, can be produced 
in mitochondrial complexes I and III in components of the tricarboxylic-acid cycle, including $\alpha$-ketoglutarate dehydrogenase (EC 1.2.4.2), and in the outter mitochondrial membrane, damaging cell components such as lipids, proteins and DNA [25, 47]. In PD, oxidative damage is a common feature, as demonstrated by increased levels of ROS in post-mortem PD brain samples [25]. Oxidative stress seems to affect various brain regions, including the $\mathrm{SN}$ and caudate nucleus, and it is accompanied by an increase in GFAP and astrocytic proliferation [50]. Additionally, PD patients present deficiencies in mitochondrial complex I in the SN, suggesting that a defect in this complex could contribute to neuronal degeneration in PD [25]. However, it is not clear whether the damage induced by ROS is a cause or a consequence of other cellular dysfunctions [25]. For example, a previous study on PD brains showed an increase in lipid peroxidation products, such as 4-hydroxinonenal, and in protein crosslinking and fragmentation [51], suggesting that oxidative stress may affect other brain regions apart from the SN.

Astrocytes produce numerous antioxidant molecules, such as GSH, catalase (EC 1.11.1.6) and SODs, providing further antioxidant protection to neurons. Unfortunately, it is known that the astrocytic protection afforded to neurons is limited, possibly due to a decline in GSH trafficking by chronic iNOS induction [52]. This depletion of GSH may facilitate the production of ROS and reactive nitrogen species (RNS) by astrocytes, causing alterations in neuronal proteins such as $\alpha$-synuclein [25]. Furthermore, the nitration of $\alpha$-synuclein by RNS can significantly enhance the synuclein fibril formation in vitro, similarly to what happens in PD brains [25]. In sum, the antioxidant properties of astrocytes have a fundamental role in the development of neurodegenerative diseases, and are considered as promising therapeutically targets.

\section{Experimental models in Parkinson}

Various pesticides, herbicides and drugs have been used in animal and in vitro models of Parkinson, as their effects mimic similar features of that seen in PD. Different epidemiological studies have shown a correlation between the exposure of these substances (especially in the case of pesticides) and appearance of PD [14-15, 17, 53]. A common feature of many of these neurotoxic compounds, such as rotenone, paraquat, or MPTP, is the inhibition of mitochondrial complex I, followed by the overproduction of ROS, ATP exhaustion, and induction of a wide range of abnormalities that can elicit neuronal and astrocytic cell death [54]. Additionally, neurotoxins induce nuclear fragmentation, endoplasmic reticulum (ER) stress and unfolded protein response in catecholaminergic cells, which are associated with changes in proteasomal and chaperone activities, similar to those observed in PD [45,55]. Other molecules used in PD models include the fungicide maneb, cyclodienes, organophosphates such as deltamethrin, DDT (dichlorodiphenyltrichloroethane), 2,4-dichlorophenoxyacetic acid, dieldrin, deguelin, diethyldithiocarbamate, paraquat, maneb, trifluralin and parathion (Figure 2) [15,56]. 


\section{Experimental Models in Parkinson Disease}

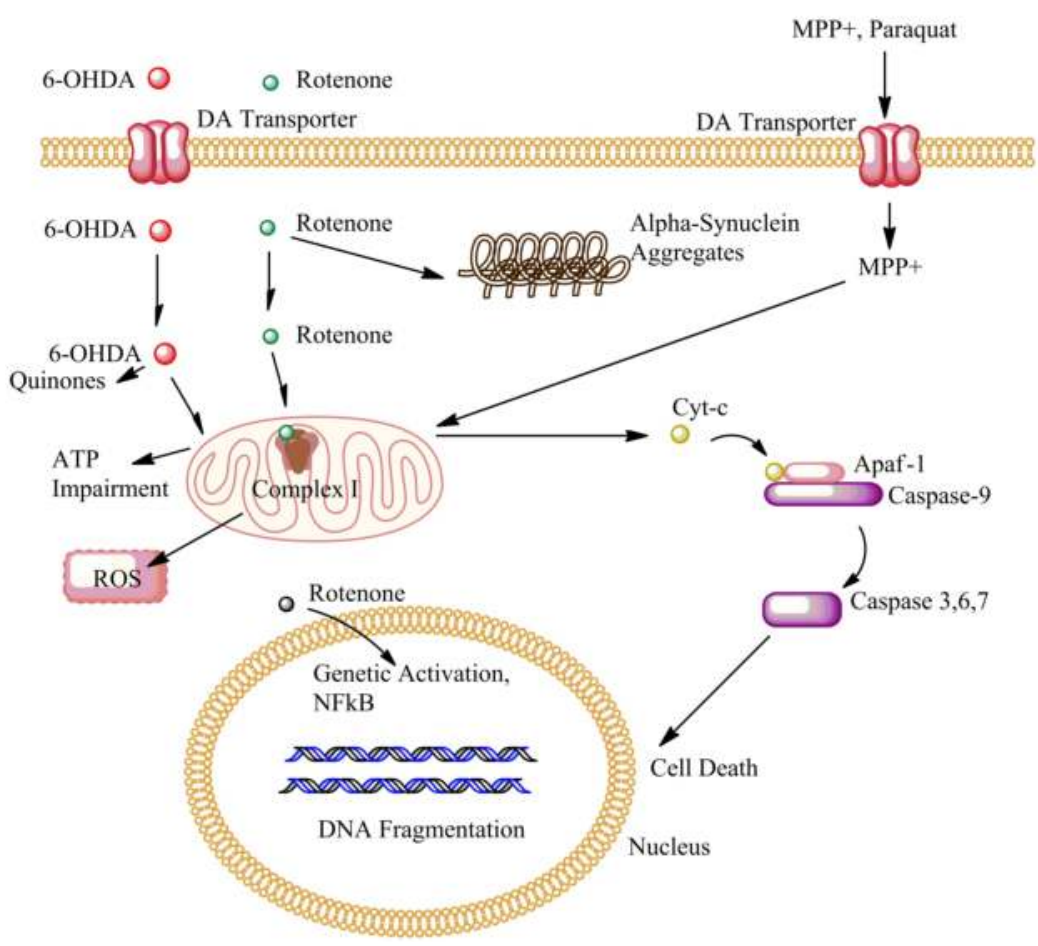

Figure 2. Experimental models in PD. Many molecules are currently used in cellular and animal models of PD, including pesticides as paraquat or rotenone and neurotoxins such as 6-OHDA and MPP+. Paraquat, 6-hydroxydopamine (6OHDA) and MPP+ easily cross cell membrane through the dopamine transporter (DA) thus inducing the formation of a-synuclein aggregates and mitochondrial impairment with the subsequent production of ROS and quinones. Compounds, as rotenone, are extremely hydrophobic and penetrate easily the cellular membrane of neurons and astrocytes. Rotenone may promote processes such as the formation of a-synuclein aggregates, and the genetic activation through the nuclear translocation of NF-KB. Additionally, as an inhibitor of mitochondrial complex I, rotenone causes the impairment of ATP, the generation of ROS and the release of proapoptotic molecules, such as cytochrome $c$ that activate caspase 9, which trigger caspases 3,6 and 7, and induce apoptosis.

\subsection{Rotenone as a Parkinson model}

Rotenone is one of the most studied neurotoxic substances used as a model for PD features and oxidative stress events in cellular and animal models [14,57]. Rotenone is a naturally occurring isoflavonoid produced in the leaves, roots and rhizomes of the tropical legumes from the genres Derris, Lonchocarpus, and Tephrosia. It is extremely hydrophobic and crosses biological membranes and serves as a high-affinity noncompetitive inhibitor of complex I, thus affecting ATP generation [58]. Rotenone is commonly used in solution as a pesticide, insecticide, or in emulsified liquid form as a piscicide $[59,60]$. 
Rotenone, and other complex I inhibitors, such as MPTP, paraquat and maneb, are used as models for assessing the environmental causes of PD [12]. Previous epidemiological studies have supported the hypothesis that a prolonged exposure to pesticides is a risk factor for PD $[17,57,61]$. Furthermore, a recent case-control study from the NIH, which reviewed 110 PD cases and 358 controls, and observed that PD incidence was increased 2.5 times in individuals who reported use of rotenone compared with nonusers [17]. Another study in agricultural workers from East Texas identified a significant increased risk $(\mathrm{OR}=10.9)$ of $\mathrm{PD}$ with the continuous use of rotenone [53]. Although these reports raised important concerns on the use of rotenone, further studies are needed to assess the detailed global epidemiology of PD by this pesticide.

Much of the research on rotenone has used animal models and different routes of administration for evaluating its effects in the Central Nervous System (CNS), especially in neurons $[14,57]$. Several groups have demonstrated that continuous systemic administration of rotenone to rats and mice reproduces key features of PD, including selective degeneration of the nigrostriatal dopaminergic system, activation of astroglia and microglia, formation of cytoplasmic inclusions in neurons, movement disorders, and defects in mitochondrial complex I [11, 14, 57, 62-64]. Previous studies have shown that intracerebral administration of rotenone damages the nigrostriatal dopaminergic pathway in rats, including the striatum fibers and neurons $[14,57]$. However, the doses employed in those experiments were much higher than the standard $\mathrm{IC}_{50}$ for rotenone. For example, doses of 2-3 mg/kg/day, similar to that reported in platelets from PD patients, produced complex I inhibition with selective nigrostriatal degeneration and astrocyte activation [14,65]. In this matter, neuronal death is thought to be a consequence of the inhibition of mitochondrial complex I, which leads to a reduction in the energy supply and subsequent collapse of the mitochondrial membrane potential [66]. A recent study suggests that rotenone administration activates caspase-2 in mice neurons inducing the activation of downstream apoptotic effectors such as Bid, Bax, caspase 3 and 9, thus initiating apoptosis [67]. Similarly, the exposure of human dopaminergic SH- SY5Y cells to rotenone caused the nuclear translocation of nuclear factor $\kappa \mathrm{B}(\mathrm{NF}-\kappa \mathrm{B})$ and the activation of caspase-3, suggesting that complex I deficiency induced by rotenone can induce NF- $\kappa \mathrm{B}-$ mediated apoptosis (Figure 3) [68].
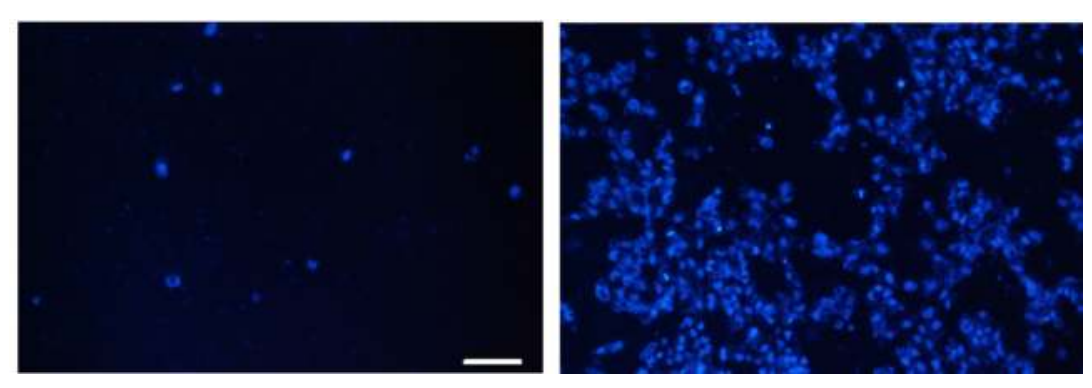

Figure 3. Rotenone-induced cell death. Astrocytic cell line ESP12 cells were treated with $30 \mathrm{nM}$ of rotenone (right) or control (right), and stained for Hoetsch 33258 to assess nuclear fragmentation. Rotenone-treated cells showed increased nuclear damage compared to controls. Scale bar, $50 \mu \mathrm{m}$. 
Alternatively, it has been postulated that rotenone-induced dopaminergic neuronal death could be dependent on the inflammatory process associated with microglial activation [64] thus indicating that rotenone differentially affects various types of CNS cells. Other previous experiments have shown that subcutaneous administration of rotenone resulted in a highly selective dopaminergic damage in neurons and $\alpha$-synuclein aggregation, similar to the Lewy bodies of PD $[63,65]$. The mechanisms by which rotenone upregulates $\alpha$-synuclein and causes its aggregation, are not well understood. A possible hypothesis is that aggregation is probably a consequence of oxidative modifications of $\alpha$-synuclein [69]. For instance, neurons and astrocytes treated with rotenone ( 25 to $50 \mathrm{nM}$ ) showed an altered expression of g-tubulin and a disorganization of the centrosome with aggregates of $\alpha$-synuclein [70]. Similarly, other studies suggest that inhibition of mitochondrial complex I activity and facilitation of $\alpha$-synuclein aggregation may be closely associated with rotenone's selective dopaminergic toxicity in neurons $[14,65]$. Furthermore, a different approach using intragastrically administered rotenone $(5 \mathrm{mg} / \mathrm{Kg}$ ) in mice showed that the accumulation and aggregation of $\alpha$-synuclein in neurons of the dorsal motor nucleus of the vagus (DMV) and the intermediolateral nucleus (IML) in the spinal cord was accompanied by the selective loss of dopaminergic neurons and astrogliosis, suggesting that the gastric administration of rotenone through the connection of the enteric nervous system (ENS) with anatomical structures of the CNS also induces PD-like features [11,19]. Rotenone has also been shown to cause increased expression of connexin43 (Cx43), which forms gap junctions, and P2X7 receptors that modulate cytokine secretion and gamma tubulin; these are important for the adequate function of the cytoskeleton and organelles such as the Golgi apparatus [70-73]. Moreover, rotenone induces astrogliosis and alterations in the expression of g-tubulin, signal transducer and activator of transcription 3 (STAT3), and connexin 43 in astrocytes [70, 72, 74].

In sum, the in vitro and in vivo evidences presented here show that dopaminergic neurons are more sensitive to rotenone toxicity than non-dopaminergic neurons, amacrine cells of retina and astrocytes [55, 75-77], possibly due to their lesser effective oxidative mechanisms and reduced supply of antioxidants [30,78]. However, astrocytes are more resilient to rotenone treatment than neurons, being its mitochondrial dysfunction tightly associated with increased neuronal death [2-4,74].

\subsection{MPTP and Parkinson}

MPTP is a widely used neurotoxicant, known for the induction of Parkinson-like symptoms such as bradikinesia, movement disorders, $\alpha$-synuclein bodies, mitochondrial abnormalities, sustained inflammation in the substantia nigra and activation of the microglia $[6,10,15,79-80]$. It was initially shown that in drug addicts, who were accidentally exposed to MPTP, there was a depletion of pigmented nerve cells in the substantia nigra, accompanied by astrogliosis and clustering of microgliosis around nerve cells [41], thus presenting some PD-like features.

MPTP is an alipophilic prototoxin that rapidly crosses the blood-brain barrier and damage dopaminergic neurons due to the selective uptake of the active metabolite $\mathrm{MPP}^{+}$via the dopamine transporter [80]. Similarly to rotenone, its neurotoxicity is induced by the inhibition of mitochondrial complex I, and subsequent energy depletion [80-81]. Additionally, $\mathrm{MPP}^{+}$has 
high affinity for noradrenergic and serotonergic uptake transporters [6,82], and its precursor, MPTP, has been mainly used in neuronal models with dopaminergic characteristics, such as the dopaminergic neuroblastoma cell line SH-SY5Y [83]. In astrocytes, MPTP has shown different (and sometimes contradictory) effects according to the experimental evidence collected in cellular and animal models. For instance, Rappold and Tieu (2010) showed that MPTP is metabolized by the astrocytic monoamineoxidase-B (MAO-B) and converted to the toxic cation $\mathrm{MPP}^{+}$, which is extruded to the extracellular space through the organic cation transporter 3 (oct3) [6,84]. Afterwards, $\mathrm{MPP}^{+}$is taken by neighboring dopaminergic neurons, thus inducing neuronal death [84]. Interestingly, silencing of oct 3 transporter in mice attenuates both the $\mathrm{MPP}^{+}$release from astrocytes and the subsequent impairment of dopaminergic neurons, in which makes oct3 as an important molecular target for dopaminergic related pathologies [6,84]. On the other hand, other authors have shown that $\mathrm{MPP}^{+}$induces negative effects in astrocytes, such as loss of viability, impairment of energetic metabolism of mitochondria, ROS generation and decrease in the glutamate clearance by astrocytes $[81,85,86]$. Taking into account the importance of MPTP, as a model for $\mathrm{PD}$, it seems that further epidemiological research is needed to address more thoroughly the role of MPTP in astrocytic damage and PD development.

\subsection{Other toxic compounds involved in Parkinson development: Paraquat and 6-OHDA}

The pesticide $N, N^{\prime}$-dimethyl-4,4'-bipyridinium dichloride (paraquat), which shares similar structure with $\mathrm{MPP}^{+}$, impairs mitochondrial functions by inducing an augmented production of oxidative stress and 4-hydroxynenal in vivo [87]. Although paraquat may not be an efficient inhibitor of mitochondrial complex I, and so does not affect dopamine uptake $[87,88]$, it does cause $\alpha$-synuclein aggregation in C57Bl/6 mice, and alters Parkin solubility, decreasing proteasome activity and causing cellular damage [87].

Paraquat has been previously shown to induce PD-like neuronal dopaminergic lesions in animal models and neuronal cell lines (Brown et al., 2006; Berry et al., 2010). Additionally, epidemiological studies suggest that long-term exposure to paraquat is associated with PD development $[15,89]$. To counteract this oxidative damage induced by paraquat, and MPTP, astrocytes seem to protect dopaminergic neurons by increasing expression of antioxidant molecules, such as heme oxigenase1 (EC 1.14.99.3), glutathione S-transferase P (EC 2.5.1.18) and glutathione [90,91]. Although this protective role of astrocytes on neuronal death by paraquat is quite promising, only few studies address this interaction and further research is needed in order to establish the precise effect of paraquat in astrocytes metabolism and neuroprotection.

Similarly to paraquat, 6-Hydroxydopamine (6-OHDA) is another widely used for in vivo and in vitro animal models of PD [92]. This compound has a structure similar to dopamine and norepinephrine and exhibits a high affinity for catecholaminergic transporters such as dopamine DAT (Dopamine transporter). 6-OHDA induces dopaminergic neuronal death by the increased generation of $\mathrm{H}_{2} \mathrm{O}_{2}$ and quinones [92]. Additionally, it causes both microgliosis and astrogliosis, which is characterized by increased astrocytic proliferation in rat cortex and striatum accompanied by a marked expression of GFAP [92,93]. Taking into account that reactive astrocytes may produce various neurotrophic factors and antioxidant mole- 
cules targeting neuronal survival, it is possible that genetic manipulation of these functions in astrocytes may represent a promising strategy to improve dopaminergic neurons or neural progenitor cells survival $[4,23]$. These neuroprotective features of astrocyte in Parkinson are further explored in the following topic.

\section{Astrocytic neuroprotection in Parkinson}

Over the last years, much research has focused on specific molecules produced by astrocytes that exert neuroprotection during brain injuries and diseases including PD, both through the reuptake of glutamate, or by producing gliotransmitters, antioxidant enzimes such as SODs, growth factors, peptide hormones and heat shock proteins [4,94-98]. Many of them have shown protective effects both in dopaminergic neurons and glial cells, and have been used in animal models and clinical trials with remarkable results (Figure 4) [31,32].

\subsection{Glutathione and Parkinson}

Astrocytes produce beneficial antioxidants, including glutathione, superoxide dismutases (SODs 1, 2 and 3), and ascorbate, which are important for neuronal survival during neurodegenerative processes [95,99-101].

The tripeptid glutathione, as the main antioxidant in the brain, is needed for the conversion of methylglyoxal, a toxic by-product of metabolism, into d-lactate by glyoxalase 1 (EC 4.4.1.5) $[94,95]$. GSH is also important in limiting and repairing the deleterious actions of $\mathrm{NO}$, but unfortunately GSH levels can be depleted by extremely high concentrations of NO [23]. For example, glutathione becomes rapidly oxidized to glutathione disulfide either by glutathione peroxidase (GPx) or by enzyme-independent chemical reactions [102]. This is an important effect against ROS formation in $\mathrm{PD}$, as it helps reducing the inhibition of complex I by NO [103]. Astrocytes possess a greater concentration of glutathione $(3,8 \mathrm{mmol} / \mathrm{L})$ than neurons $(2.5 \mathrm{mmol})$, probably due to a higher content of the astrocytic enzyme y-glutamylcysteine synthethase (EC 6.3.2.2) [6]. For example, neurons co-cultured with astrocytes exhibit higher levels of glutathione compared to neurons cultured alone, demonstrating that astrocytes provide additional antioxidant defenses to neurons [104-106]. Additionally, an increase in glutathione peroxidase-containing cells shows to be inversely correlated with the severity of dopaminergic cell loss in cell populations from patients with PD, suggesting that the quantity of glutathione peroxidase in cells might be critical for a protective effect against oxidative stress during PD [107].

The greater production of GSH by astrocytes seems to be dependent on the preferential activation of transcription factor Nrf2 in astrocytes, which leads to a more efficient GSH synthesis and higher GSH content in astrocytes than in neurons [108]. Interestingly, Nrf2 is known to regulate the expression of cytoprotective genes, and factors essential to neuronal survival [6,108]. Additionally, Nrf2 knockout mice are more sensitive to mitochondrial complex inhibitors such as MPTP and 3-nitropropionic acid [108], suggesting an important role of this transcription factor in scavenging free radicals. On the other hand, decrease in glutathione is 
one of the earliest biochemical changes in PD and incidental Lewy body disease [109]. Additionally, the GSH content was significantly reduced in the substantia nigra of PD patients, suggesting that GSH depletion enhances neuronal death under certain pathological conditions [6]. Interestingly, this evidence is consistent with the data in PD patients, in which glutathione-containing cells are in regions with preserved dopaminergic neurons [52].
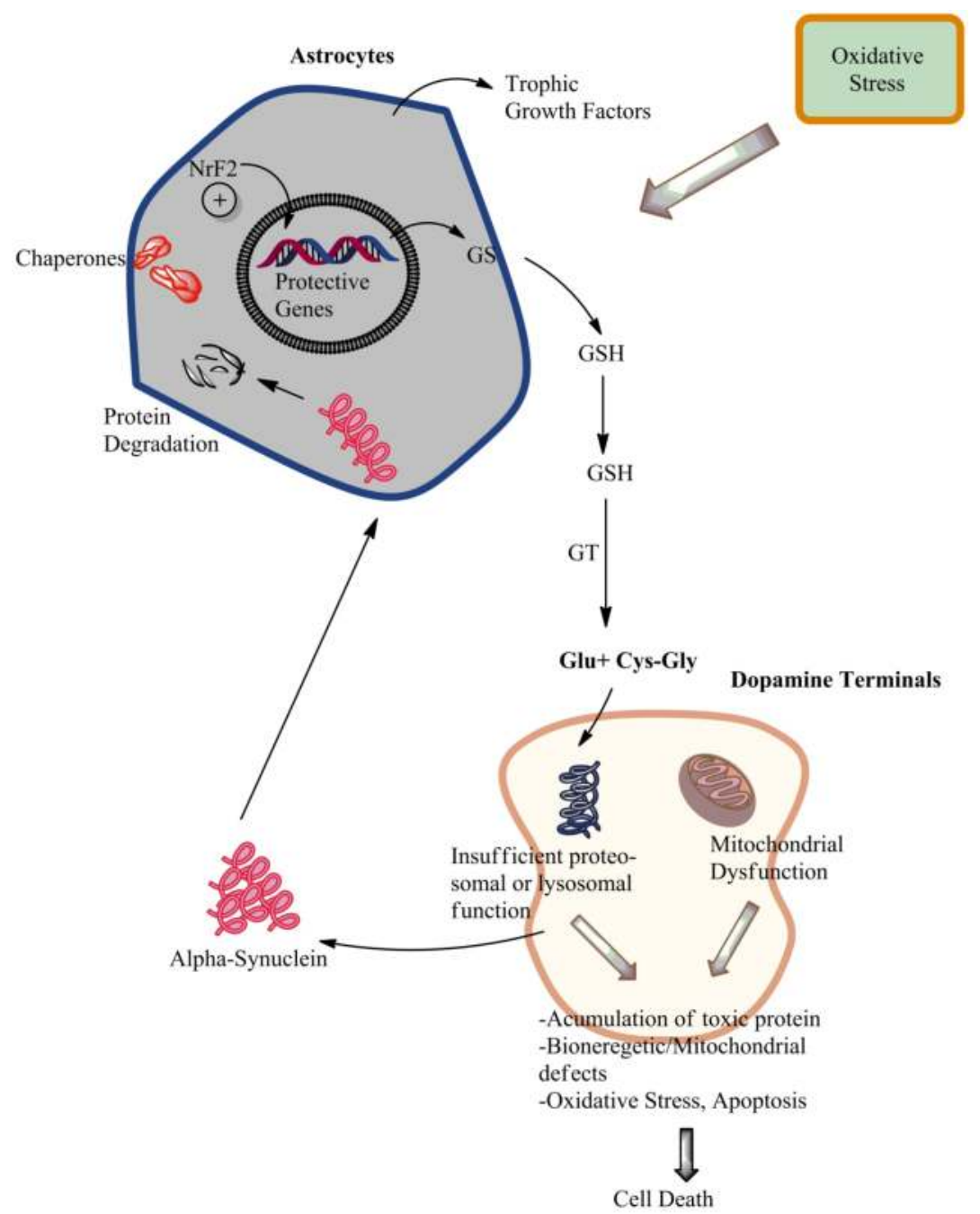

Figure 4. Astrocytes-mediate neuroprotection through multiple signaling pathways Astrocytes release glutathione, which serves as precursors for neuronal GSH synthesis, and trophic growth factors such as bFGF, GDNF, and MANF. Activation of the transcription factor $\mathrm{Nrf2}$ leads to the expression of antioxidant genes, including $\gamma$-glutamylcysteine synthetase (GS), which is involved in GSH synthesis and removal or degradation of cytotoxic molecules, such as a-synuclein. 
It is possible that the recovery of glutathione levels may enhance the survival of affected neurons, either by increasing synthesis of GSH or by slowing its degradation [25]. However, the GSH blood-brain barrier permeability is low, and clinical trials using injections of GSH have shown little benefits $[6,25,110]$. Alternatively, it has been demonstrated that the use of GSH precursors, such as glutamyl cysteine ethyl ester (GCEE) and gluthathione ethyl ester (GEE), increases significantly the intracellular glutathione levels in neuronal cells, protecting dopaminergic neurons against oxidative an nitrosative stress, both in animal and cellular models [25,109]. Finally, the modulation of Nrf2 downstream signaling may be considered as a promising strategy for enhancing the astrocytic production of GSH [108], which may counteract the oxidative imbalance that likely affects neurons in neurodegenerative processes such as PD.

\subsection{Superoxide dismutases and Parkinson}

Superoxide dismutases catalyze the dismutation of superoxide ions into oxygen and hydrogen peroxide [23]. As such, they are an important antioxidant defense in nearly all cells exposed to oxygen. In most mammalian cells, SOD is present in three isoforms: a cytosolic copper, zinc superoxide dismutase (SOD1); a mitochondrial manganese superoxide dismutase (SOD2); and an extracellular copper, zinc superoxide dismutase (SOD3) $[23,112]$. Given its importance in neuroprotection, SODs and other antioxidant molecules released by astrocytes are highly studied in neurodegenerative diseases like PD and in other oxidative-related events. Evidence that SODs defend against oxidative stress in situ has been obtained using transgenic mutants that either overexpress or lack these antioxidant enzymes [111]. For example, the overexpression of $\mathrm{Cu} / \mathrm{Zn}$ SOD was able to rescue dopaminergic neurons and diminishes locomotor disabilities in a Drosophila mutant model for $\alpha$-synuclein overexpression [112]. Interestingly in PD patients, it has been shown, an specific increase in SOD levels in the substantia nigra, with no changes in activities of glutathione peroxidase, catalase and glutathione reductase (EC 1.8.1.7) [25]. A similar increase was observed in the mitochondrial isoform of SOD in the motor cortex from PD patients [113], suggesting that SODs have a greater importance than other antioxidant enzymes during PD development. Further research is needed in order to address the therapeutic application of SOD in PD and other diseases.

\subsection{Astrocytic chaperones and Parkinson}

Chaperones belonging to the conserved family of Heat shock proteins (Hsps) are proteins involved in the regulation of protein folding, translocation of proteins across membranes, regulation of cell death and assembling of protein [114]. Interestingly, protein aggregates, and misfolded proteins have been found in AD, Huntington, PD, prion disease, ALS and other neurological injuries [115-117]. Furthermore, previous evidence suggests that formation of unfolded proteins in astrocytes could induce the inflammatory responses previously mentioned [117].

Many Hsps are currently being considered for the potential treatment of diseases involving protein aggregation and misfolding such as the case of PD [116,118]. These include the chap- 
erones, DJ-1, Hsp70, Hsp9- and the co-chaperone Hsp40, and members from the Bag family, such as Bag 5, CHIP and suppression of tumorigenicity 13 (ST13) [118]. Several of these chaperones are colocalized or associated with the PD related proteins, E3-ubiquitin ligase (E 6.3.2.19), parkin, $\alpha$-synuclein and the dopamine transporter (DAT) [119].

DJ-1, also known as PARK7, is upregulated in reactive astrocytes and serves as a redox-sensitive chaperone with antiapoptotic properties [119]. DJ-1, both in normal and mutant forms, colocalizes with Hsp70 and CHIP in the cytosol. Following oxidative stress, this molecule is translocated to the mitochondria, where it becames associated with the chaperone GRP75 $[119,120]$. It has been previously shown that DJ-1 can suppress the aggregation and oligomerization of $\alpha$-synuclein, thus promoting its degradation, which is dependent on the redox state of the cell environment $[119,121]$. Additionally, DJ-1 regulates signaling pathways such P38 mitogen-activated protein kinases (MAPK; EC 2.7.11.24), apoptosis signal-regulating kinase 1 (ASK1; EC 2.7.11.25) and protein kinase B (AKT) following cellular production of ROS, suggesting that this chaperone is an important redox-reactive molecule during oxidative stress in PD and other age-related disorders [120].

Hsp70 family of chaperones are thought to be critical in the regulation of protein oligomerization and aggregation, which are believed to be central in the molecular pathogenesis of PD and other neurodegenerative diseases [118]. For example, the overexpression of Hsp70 has been found to protect PC12 cells, and dopaminergic neurons against MPTP toxicity [118,119]. Additionally, the overexpression of Hsp70 in mice has been shown to reduce the amount of misfolded and aggregated $\alpha$-synuclein species, suggesting a protection of this chaperone against $\alpha$ synuclein-dependent toxicity [122]. It seems that $\alpha$-synuclein degradation mediated by Hsp70 occurs in the proteasome or in the lisosomes by a selective process called chaperone-mediated autophagy (CMA) [114]. The wild type, but not a mutant form of $\alpha$-synuclein is degradated by CMA, suggesting that this mechanism is important in the formation of $\alpha$-synuclein aggregates during PD [114]. Importantly, the astrocytic clearance of $\alpha$-synuclein by chaperones, like Hsp70, may confer additional neuroprotection to dopaminergic neurons [6,114].

Chaperones located in other organelles, such as the ER, have also been studied in the development of neurodegenerative processes. For example, homocysteine-induced endoplasmic reticulum protein, which is located in the ER membrane of neurons and astrocytes in the Central Nervous System (SNC), is found accumulated in Lewy bodies, suggesting a role in their formation and further development of PD [117]. In sum, given the central importance of chaperones in protein homeostasis, or proteostasis, they may serve as rational targets for the design of therapeutic strategies in neurodegenerative diseases associated with aberrant protein folding including PD.

\subsection{Growth factors and Parkinson}

Several neurotrophic and growth factors have been shown to protect dopaminergic neurons and glial cells against induced excitotoxicity by the activation of specific signaling pathways that are responsible for cell survival and axonal sprouting [31,32]. Some of them have also been tested in PD clinical trials with some promising results [31,32]. For example, brain derived neurotrophic factor (BDNF) and TNF protect neurons against excitotoxicity through 
activation of the transcription factor NF-kB, which induces the expression of antioxidant enzymes such as Mn-SOD and the anti-apoptotic proteins, Bcl-2 and inhibitor of apoptosis proteins IAPs [123,124]. Additionally, the endogenous administration of BDNF was shown to protect neurons within the substantia nigra from 6-OHDA and MPTP toxicity, both in rat and primate Parkinson models [31].

The family of glial cell line-derived neurotrophic factor (GDNF) comprises ligands such as GDNF, neurturin (NRTN), artemin (ARTN) and persephin. GDNF, secreted by astrocytes, is essential for the survival of dopaminergic neurons [32]. It has been shown that GDNF administration by catheter increases dopaminergic neuronal resistance against 6-OHDA toxicity, but with preservation of motor functions in rat and rhesus monkey models [96]. However, clinical trials in patients that were administered GDNF in different regions of the brain have shown mixed results and further research is needed [31, 125-127].

Insulin-like growth factors (IGFs) signaling through the phosphatidylinositol 3-kinase (PI3K/Akt) downstream pathway can protect neurons against LPS excitotoxicity in cell culture and in vivo [124, 128,129]. Furthermore, the activation of this signaling pathway by IGFI can suppress $\alpha$-synuclein aggregation and toxicity, suggesting a possible therapeutically strategy in PD [130]. Similarly to IGF-I, vascular endothelial growth factor (VEGF) affects the survival and proliferation of endothelial cells, neurons and astrocytes in the brain, suggesting a potential therapeutic application in PD [32]. Additionally, VEGF-B (isorform B) was found activated in a rat brain model following treatment with $40 \mathrm{nM}$ rotenone, and showed neuroprotective actions by improving neuronal survival (Falk et al., 2009). Some studies suggest that VEGF promotes neuroprotection by signaling through the neuropilin receptor expressed in DA neurons, and indirectly by activating the proliferation of astroglia and by promoting angiogenesis [32,131,132]. Furthermore, the striatal injection of an adenoassociated virus (AAV)-mediated VEGF expression provided neuroprotection and behavioral improvement in rats treated with 6-OHDA [133].

Basic fibroblast growth factor (bFGF) protects hippocampal and cortical neurons against glutamate toxicity by changing the expression of $N$-methyl-D-aspartic acid (NMDA) receptors and antioxidant enzymes like superoxide dismutases and glutathione reductase [124]. Furthermore, a coculture of transgenic overexpressing FGF-2 Schwann cells with dopaminergic neurons improved the survival of dopaminergic neurons and the behavioral outcome in a parkinsonian rat model lesioned with 6-OHDA [134]. Finally, there are other neurotrophic factors that have shown dopaminergical neuronal protection in Parkinson-like models, including hepatocyte growth factor (HGF), mesencephalic astrocyte-derived neurotrophic factor (MANF), cerebral dopaminergic neurotrophic factor (CDNF), granulocyte colonystimulating factor (G-CSF), and platelet derived growth factor (PDGF-CC) [31-32, 135-136].

\section{Conclusions and future perspectives}

In recent years a growing body of evidence has demonstrated that the malfunctioning of astrocytes may contribute to various neurodegenerative diseases, including Alzheimer, ALS, 
multiple sclerosis, and Parkinson. Importantly, astrocytes are involved in both exacerbation of damage and in neuroprotective mechanisms that are crucial for neuronal survival. In this matter, astrocytes are essential for the regulation of oxidative stress and ROS production, both in normal and in pathological circumstances.

The overexpression of antioxidant molecules such as GSH and SOD2, or chaperones such as Hsp70 has proved to be a successful experimental approach in brain diseases, including PD. The use of growth factors, both in animal models and in clinical trials, has shown promising effects in protecting dopaminergic neurons and astrocytes in damaged regions by the activation of different signaling pathways important in neuronal survival and regeneration. It is important to mention that mitochondrial protection in astrocytes is an important asset to maintain the energetic balance of the brain and the antioxidant production that contribute to neuronal protection. Therefore future efforts in neuroprotective strategies should emphasize the mitochondrial protection in astrocytes. Finally, the combination of novel drug therapies, a better understanding of the $\alpha$-synuclein clearance by astrocytes, the use of neurotoxic models, growth factors use and other therapies that increase astrocyte survival and its antioxidant function may shed light on a prospective cure of PD in the near future.

\section{Acknowledgements}

This work was supported in part by grants PUJ IDs 4509 and 4327 to GEB.

\section{Author details}

Ricardo Cabezas ${ }^{1}$, Marco Fidel Avila ${ }^{1}$, Daniel Torrente ${ }^{1}$, Ramon Santos El-Bachá ${ }^{2}$ Ludis Morales $^{1}$, Janneth Gonzalez ${ }^{1}$ and George E. Barreto ${ }^{1}$

*Address all correspondence to: gsampaio@javeriana.edu.co

1 Departamento de Nutrición y Bioquímica, Facultad de Ciencias, Pontificia Universidad Javeriana, Bogotá D.C., Colombia

2 Laboratorio de Neuroquímica e Biologia Celular, Universidade Federal da Bahía, Salvador, Bahia, Brazil

\section{References}

[1] Fernandez HH. Updates in the medical management of Parkinson disease. Cleveland Clinic Journal of Medicine 2012; 79(1) 28-35. 
[2] Volterra A, Meldolesi J. Astrocytes, from brain glue to communication elements: the revolution continues. Nature Reviews Neuroscience 2005; 6(8) 626-640.

[3] Hamby ME, Sofroniew MV. Reactive astrocytes as therapeutic targets for CNS disorders. Neurotherapeutics 2010; 7(4) 494-506.

[4] Barreto GE, Gonzalez J, Capani F, Morales L. Role of Astrocytes in Neurodegenerative Diseases. In: Raymond Chuen-Chung Chang. (ed.) Neurodegenerative Diseases Processes, Prevention, Protection and Monitoring. InTech; 2011. p257-272.

[5] Nutt JG, Wooten GF. Clinical practice. Diagnosis and initial management of Parkinson's disease. The New England Journal of Medicine 2005; 353(10) 1021-1027.

[6] Rappold PM, Tieu K. Astrocytes and therapeutics for Parkinson's disease. Neurotherapeutics 2010; 7(4) 413-423.

[7] Halliday GM, Stevens CH. Glia: initiators and progressors of pathology in Parkinson's disease. Movement Disorders 2011; 26(1) 6-17.

[8] Singer C. Managing the patient with newly diagnosed Parkinson disease. Cleveland Clinic Journal of Medicine 2012; 79(Suppl 2) S3-S7.

[9] Schwartz MD, Sabetay MD. An Approach to the continuous Dopaminergic Stimulation in Parkinson's Disease. The Israel Medical Association Journal 2012; 14(3) 175-179

[10] Hirsch EC, Breidert T, Rousselet E, Hunot S, Hartmann A, Michel PP. The role of glial reaction and inflammation in Parkinson's disease. Annals of the New York Academy of Sciences 2003; 991 214-228.

[11] Pan-Montojo F, Anichtchik O, Dening Y, Knels L, Pursche S, Jung R, et al. Progression of Parkinson's disease pathology is reproduced by intragastric administration of rotenone in mice. PLoS One 2010; 5 (1) e8762

[12] Wang HL, Chou AH, Wu AS, Chen SY, Weng YH, Kao YC, et al. PARK6 PINK1 mutants are defective in maintaining mitochondrial membrane potential and inhibiting ROS formation of substantia nigra dopaminergic neurons. Biochimica et Biophysica Acta 2011; 1812(6) 674-684.

[13] Knott AB, Bossy-Wetzel E. Impairing the mitochondrial fission and fusion balance: a new mechanism of neurodegeneration. Annals of the New York Academy of Sciences 2008; 1147 283-292.

[14] Betarbet R, Sherer TB, MacKenzie G, Garcia-Osuna M, Panov, AV, Greenamyre, JT. Chronic systemic pesticide exposure reproduces features of Parkinson's disease. Nature Neuroscience 2000; 3(12) 1301-1306.

[15] Brown TP, Rumsby PC, Capleton AC, Rushton L, Levy LS. Pesticides and Parkinson's disease -- is there a link? Environmental Health Perspectives 2006; 114(2) 156-64. 
[16] Greenamyre JT, Betarbet R, Sherer TB. The rotenone model of Parkinson's disease: genes, environment and mitochondria. Parkinsonism \& Related Disorders 2003; 9 (Suppl 2) S59-S64.

[17] Tanner CM, Kamel F, Ross GW, Hoppin JA, Goldman SM, Korell M, et al. Rotenone, paraquat, and Parkinson's disease. Environmental Health Perspective 2011; 119(6) 866-872.

[18] Vives-Bauza C, Przedborski S. Mitophagy: the latest problem for Parkinson's disease. Trends in Molecular Medicine 2011; 17(3) 158-165.

[19] Braak H, de Vos RA, Bohl J, Del Tredici K. Gastric alph $\alpha$-synuclein immunoreactive inclusions in Meissner's and Auerbach's plexuses in cases staged for Parkinson's disease-related brain pathology. Neuroscience Letters 2006; 396(1) 67-72.

[20] Wakabayashi K, Hayashi S, Yoshimoto M, Kudo H, Takahashi H. NACP/alph $\alpha$-synuclein-positive filamentous inclusions in astrocytes and oligodendrocytes of Parkinson's disease brains. Acta Neuropathologica 2000; 99(1) 14-20.

[21] Ciesielska A, Joniec I, Kurkowska-Jastrzebska I, Cudna A, Przybylkowski A, Czlonkowska A, et al. The impact of age and gender on the striatal astrocytes activation in murine model of Parkinson's disease. Inflammation Research 2009; 58(11) 747-753.

[22] Muramatsu Y, Kurosaki R, Watanabe H, Michimata M, Matsubara M, Imai Y, et al. Cerebral alterations in a MPTP-mouse model of Parkinson's disease--an immunocytochemical study. The Journal of Neural Transmission 2003; 110(10) 1129-1144.

[23] Chen Y, Swanson RA. Astrocytes and brain injury. Journal of Cerebral Blood Flow \& Metabolism 2003; 23(2) 137-149.

[24] Maragakis NJ, Rothstein JD. Mechanisms of Disease: astrocytes in neurodegenerative disease. Nature Clinical Practice Neurology 2006; 2(12) 679-689.

[25] Chinta S, Andersen J. Redox imbalance in Parkinson. Biochimica et Biophysica Acta 2008; 1780(11) 1362-1367.

[26] Kimelberg HK, Nedergaard M. Functions of astrocytes and their potential as therapeutic targets. Neurotherapeutics 2010; 7(4) 338-353.

[27] Parpura V, Grubisic V, Verkhratsky A. Ca(2+) sources for the exocytotic release of glutamate from astrocytes. Biochimica et Biophysica Acta 2011; 1813(5) 984-991.

[28] Bushong EA, Martone ME, Jones YZ, Ellisman MH. Protoplasmic astrocytes in CA1 stratum radiatum occupy separate anatomical domains. The Journal of Neuroscience 2002; 22(1) 183-192.

[29] Zonta M, Angulo MC, Gobbo S, Rosengarten B, Hossmann KA, Pozzan T, et al. Neuron-to-astrocyte signaling is central to the dynamic control of brain microcirculation. Nature Neuroscience 2003; 6(1) 43-50. 
[30] Greve MW, Zink BJ. Pathophysiology of traumatic brain injury. Mount Sinai Journal of Medicine 2009; 76(2) 97-104.

[31] Ramaswamy S, Kordower JH. 2009. Are growth factors the answer? Parkinsonism and Related Disorders 2009; 15(Suppl 3) S176-S180.

[32] Yasuda T, Mochizuki H. Use of growth factors for the treatment of Parkinson's disease. Expert Review of Neurotherapeutics 2010; 10(6) 915-924.

[33] Barreto G, Veiga S, Azcoitia I, Garcia-Segura LM, Garcia-Ovejero D. Testosterone decreases reactive astroglia and reactive microglia after brain injury in male rats: role of its metabolites, oestradiol and dihydrotestosterone. European Journal of Neuroscience 2007; 25(10) 3039-3046.

[34] Barreto G, Santos-Galindo M, Diz-Chaves Y, Pernía O, Carrero P, Azcoitia I, GarciaSegura LM. Selective estrogen receptor modulators decrease reactive astrogliosis in the injured brain: effects of aging and prolonged depletion of ovarian hormones. Endocrinology 2009; 150(11) 5010-5015.

[35] Gu XL, Long CX, Sun L, Xie C, Lin X, Cai H. Astrocytic expression of Parkinson's disease-related A53T alph $\alpha$-synuclein causes neurodegeneration in mice. Molecular Brain 2010; 21(3) 12.

[36] Xiong X, Barreto GE, Xu L, Ouyang YB, Xie X, Giffard RG. Increased brain injury and worsened neurological outcome in interleukin-4 knockout mice after transient focal cerebral ischemia. Stroke 2011; 42(7) 2026-2032.

[37] Adelson JD, Barreto GE, Xu L, Kim T, Brott BK, Ouyang YB, et al. Neuroprotection from stroke in the absence of MHCI or PirB. Neuron 2012; 73(6) 1100-1107.

[38] Ridet, JL, Malhotra SK, Privat A, Gage FH. Reactive astrocytes: cellular and molecular cues to biological function. Trends in Neurosciences 1997; 20(12) 570-577.

[39] Sugaya K, Chou S, Xu SJ, McKinney M. Indicators of glial activation and brain oxidative stress after intraventricular infusion of endotoxin. Brain Research Molecular Brain Research 1998; 58(1-2) 1-9.

[40] Kang W, Hebert JM. Signaling pathways in reactive astrocytes, a genetic perspective. Molecular Neurobiology 2011; 43(3) 147-154.

[41] Langston JW, Forno LS, Tetrud J, Reeves AG, Kaplan JA, Karluk D. Evidence of active nerve cell degeneration in the substantia nigra of humans years after 1-methyl-4phenyl-1,2,3,6-tetrahydropyridine exposure. Annals of Neurology 1999; 46(4) 598-605.

[42] Herrera AJ, Castaño A, Venero JL, Cano J, Machado A. The single intranigral injection of LPS as a new model for studying the selective effects of inflammatory reactions on dopaminergic system. Neurobiology of Disease 2000; 7(4) 429-447. 
[43] Niranjan R, Nath C, Shukla R. The mechanism of action of MPTP-induced neuroinflammation and its modulation by melatonin in rat astrocytoma cells, C6. Free Radical Research 2010; 44(11) 1304-1316.

[44] Samantaray S, Knaryan VH, Guyton MK, Matzelle DD, Ray SK, Banik NL. The parkinsonian neurotoxin rotenone activates calpain and caspase-3 leading to motoneuron degeneration in spinal cord of Lewis rats. Neuroscience 2007; 146(2) 741-755.

[45] de Oliveira DM, Barreto G, Galeano P, Romero JI, Holubiec MI, Badorrey MS, et al. Paullinia cupana Mart. var. Sorbilis protects human dopaminergic neuroblastoma SH-SY5Y cell line against rotenone-induced cytotoxicity. Human \& Experimental Toxicology 2011; 30(9) 1382-1391.

[46] Cherian L, Goodman JC, Robertson CS. Brain nitric oxide changes after controlled cortical impact injury in rats. Journal of Neurophysiology 2000; 83(4) 2171-2178.

[47] LeDoux SP, Druzhyna NM, Hollensworth SB, Harrison JF, Wilson GL. Mitochondrial DNA repair: a critical player in the response of cells of the CNS to genotoxic insults. Neuroscience 2007; 145(4) 1249-1259.

[48] Dugan LL, Kim-Han JS. Astrocyte mitochondria in in vitro models of ischemia. Journal of Bioenergetics and Biomembranes 2004; 36(4) 317-321.

[49] Patel VP, Chu CT. Nuclear transport, oxidative stress, and neurodegeneration. International Journal of Clinical and Experimental Pathology 2011; 4(3) 215-229.

[50] Mythri RB, Venkateshappa C, Harish G, Mahadevan A, Muthane UB, Yasha TC, et al. Evaluation of markers of oxidative stress, antioxidant function and astrocytic proliferation in the striatum and frontal cortex of Parkinson's disease brains. Neurochemical Research 2011;36(8) 1452-1463.

[51] Jenner P. Oxidative stress in Parkinson's disease. Annals of Neurology 2003; 53 S26S36.

[52] Heales SJ, Lam AA, Duncan AJ, Land JM. Neurodegeneration or neuroprotection: the pivotal role of astrocytes. Neurochemical Research 2004; 29(3) 513-519.

[53] Dhillon AS, Tarbutton GL, Levin JL, Plotkin GM, Lowry LK, Nalbone JT, et al. Pesticide/environmental exposures and Parkinson's disease in East Texas. Journal of Agromedicine 2008; 13(1) 37-48.

[54] Dick FD. Parkinson's disease and pesticide exposures. British Medical Bulletin 2006; 79-80 219-231.

[55] Nagel F, Bahr M, Dietz GP. Tyrosine hydroxylase-positive amacrine interneurons in the mouse retina are resistant against the application of various parkinsonian toxins. Brain Research Bulletin 2009; 79(5) 303-309. 
[56] Caboni P, Sherer TB, Zhang N, Taylor G, Na HM, Greenamyre JT, et al. Rotenone, deguelin, their metabolites, and the rat model of Parkinson's disease. Chemical Research in Toxicology 2004; 17(11) 1540-1548.

[57] Liu B, Gao HM, Hong JS. Parkinson's disease and exposure to infectious agents and pesticides and the occurrence of brain injuries: role of neuroinflammation. Environmental Health Perspectives 2003; 111(8) 1065-1073.

[58] Greenamyre JT, MacKenzie G, Peng TI, Stephans SE. Mitochondrial dysfunction in Parkinson's disease. Biochemical Society Symposia 1999; 66 85-97

[59] Isman MB. Botanical insecticides, deterrents, and repellents in modern agriculture and an increasingly regulated world. Annual Review of Entomology 2006; 51 45-66.

[60] Patel F. Pesticidal suicide: adult fatal rotenone poisoning. Journal of Forensic and Legal Medicine 2011; 18(7) 340-342.

[61] Tuchsen F, Jensen AA. Agricultural work and the risk of Parkinson's disease in Denmark, 1981-1993. Scandinavian Journal of Work, Environment \& Health 2000; 26(4) 359-362.

[62] Greenamyre JT, Cannon JR, Drolet R, Mastroberardino PG. Lessons from the rotenone model of Parkinson's disease. Trends in Pharmacological Science 2010; 31(4) 141-142.

[63] Hoglinger GU, Lannuzel A, Khondiker ME, Michel PP, Duyckaerts C, Feger J, et al. The mitochondrial complex I inhibitor rotenone triggers a cerebral tauopathy. Journal of Neurochemistry 2005; 95(4) 930-939.

[64] Ogawa N, Asanuma M, Miyazaki I, Diaz-Corrales FJ, Miyoshi K. L-DOPA treatment from the viewpoint of neuroprotection. Possible mechanism of specific and progressive dopaminergic neuronal death in Parkinson's disease. Journal of Neurology 2005; 252 (Suppl 4) IV23-IV31.

[65] Sherer TB, Betarbet R, Kim JH, Greenamyre JT. Selective microglial activation in the rat rotenone model of Parkinson's disease. Neuroscience Letters 2003; 341(2) 87-90.

[66] Gyulkhandanyan AV, Feeney CJ, Pennefather PS. Modulation of mitochondrial membrane potential and reactive oxygen species production by copper in astrocytes. Journal of Neurochemistry 2003; 87(2) 448-460.

[67] Tiwari M, Lopez-Cruzan M, Morgan WW, Herman B. Loss of caspase-2-dependent apoptosis induces autophagy after mitochondrial oxidative stress in primary cultures of young adult cortical neurons. The Journal of Biological Chemistry 2011; 286(10) 8493-8506.

[68] Wang HL, Chou AH, Wu AS, Chen SY, Weng YH, Kao YC, et al. PARK6 PINK1 mutants are defective in maintaining mitochondrial membrane potential and inhibiting 
ROS formation of substantia nigra dopaminergic neurons. Biochimica et Biophysica Acta 2011; 1812(6) 674-684.

[69] Greenamyre JT, Betarbet R, Sherer TB. The rotenone model of Parkinson's disease: genes, environment and mitochondria. Parkinsonism \& Related Disorders 2003; 9 (Suppl 2) S59-S64.

[70] Diaz-Corrales FJ, Asanuma M, Miyazaki I, Miyoshi K, Ogawa N. Rotenone induces aggregation of gamma-tubulin protein and subsequent disorganization of the centrosome: relevance to formation of inclusion bodies and neurodegeneration. Neuroscience 2005; 133(1) 117-135.

[71] Kawasaki A, Hayashi T, Nakachi K, Trosko JE, Sugihara K, Kotake Y, et al. Modulation of connexin 43 in rotenone-induced model of Parkinson's disease. Neuroscience 2009; 160(1) 61-68.

[72] Zhang S, Liang R, Zhou F, Huang X, Ding JH, Hu, G. Reversal of rotenone-induced dysfunction of astrocytic connexin 43 by opening mitochondrial ATP-sensitive potassium channels. Cellular and Molecular Neurobiology 2010; 31(1) 111-117.

[73] Gao XF, Wang W, Yu Q, Burnstock G, Xiang ZH. He C. Astroglial P2X7 receptor current density increased following long-term exposure to rotenone. Purinergic Signalling 2011; 7(1) 65-72.

[74] Sarafian TA, Montes C, Imura T, Qi J, Coppola G, Geschwind DH, et al. Disruption of astrocyte STAT3 signaling decreases mitochondrial function and increases oxidative stress in vitro. PloS One 2010; 5(3) e9532.

[75] Ahmadi FA, Grammatopoulos TN, Poczobutt AM, Jones SM, Snell LD, Das M, et al. 2008. Dopamine selectively sensitizes dopaminergic neurons to rotenone-induced apoptosis. Neurochemical Research 2008; 33(5) 886-901.

[76] Norazit A, Meedeniya AC, Nguyen MN, Mackay-Sim A. Progressive loss of dopaminergic neurons induced by unilateral rotenone infusion into the medial forebrain bundle. Brain Research 2010; 1360, 119-129.

[77] Radad K, Gille G, Rausch WD. Dopaminergic neurons are preferentially sensitive to long-term rotenone toxicity in primary cell culture. Toxicology In Vitro 2008; 22(1) 68-74.

[78] Verkhratsky A, Rodriguez JJ, Parpura V. Calcium signalling in astroglia. Molecular and Cellular Endocrinology 2012; 353(1-2) 45-56.

[79] McGeer PL, McGeer EG. Glial reactions in Parkinson's disease. Movement Disorders 2008; 23(4) 474-83.

[80] Sonsalla PK, Zeevalk GD, German DC. Chronic intraventricular administration of 1methyl-4-phenylpyridinium as a progressive model of Parkinson's disease. Parkinsonism \& Related Disorders 2008; 14( Suppl 2): S116-S118. 
[81] Di Monte, DA, Tokar I, Langston JW, Impaired glutamate clearance as a consequence of energy failure caused by $\mathrm{MPP}(+)$ in astrocytic cultures. Toxicology and Applied Pharmacology 1999; 158(3) 296-302.

[82] Javitch JA, D'Amato RJ, Strittmatter SM, Snyder SH. Parkinsonism-inducing neurotoxin, $N$-methyl-4-phenyl-1,2,3,6-tetrahydropyridine:uptake of the metabolite $N$ methyl-4-phenylpyridine by dopamine neurons explain selective toxicity. Proceedings of the National Academy of Sciences of the United States of America 1985; 82(7) 2173-2177.

[83] Xie HR, Hu LS, Li GY. SH-SY5Y human neuroblastoma cell line: in vitro cell model of dopaminergic neurons in Parkinson's disease. Chinese Medical Journal 2010; 123(8) 1086-1092.

[84] Cui M, Aras R, Christian WV, Rappold PM, Hatwar M, Panza J, et al. The organic cation transporter-3 is a pivotal modulator of neurodegeneration in the nigrostriatal dopaminergic pathway. Proceedings of the National Academy of Sciences of the United States of America 2009; 106(19) 8043- 8048.

[85] Di Monte DA, Wu EY, Delanney LE, Irwin I, Langston JW. Toxicity of 1-methyl-4phenyl-1,2,3,6-tetrahydropyridine in primary cultures of mouse astrocytes. The Journal of Pharmacology and Experimental Therapeutics 1992; 261(1) 44-49.

[86] Bi J, Wang XB, Chen L, Hao S, An LJ, Jiang B, et al. Catalpol protects mesencephalic neurons against MPTP induced neurotoxicity via attenuation of mitochondrial dysfunction and MAO-B activity. Toxicology in Vitro 2008; 22(8) 1883-1889.

[87] Berry C, La Vecchia C, Nicotera P. Paraquat and Parkinson's disease. Cell Death and Differentiation 2010; 17(7) 1115-1125.

[88] Richardson JR, Quan Y, Sherer TB, Greenamyre JT, Miller GW. Paraquat neurotoxicity is distinct from that of MPTP and rotenone. Toxicological Sciences 2005; 88(1) 193201.

[89] Liou HH, Tsai MC, Chen CJ, Jeng JS, Chang YC, Chen SY, et al. Environmental risk factors and Parkinson's disease: a case-control study in Taiwan. Neurology 1997; 48(6) 1583-1588.

[90] Olesen BT, Clausen J, Vang O. Characterization of the transcriptional profile in primary astrocytes after oxidative stress induced by Paraquat. Neurotoxicology 2008; 29(1) 13-21.

[91] Rathinam ML, Watts LT, Narasimhan M, Riar AK, Mahimainathan L, Henderson GI. Astrocyte mediated protection of fetal cerebral cortical neurons from rotenone and paraquat. Environmental toxicology and pharmacology 2012; 33(2) 353-60.

[92] Bové J, Prou D, Perier C, Przedborski S Toxin-induced models of Parkinson's disease. NeuroRX: the journal of the American society for experimental Neurotherapeutics 2005; 2(3) 484-494. 
[93] Wachter B, Schurger S, Rolinger J, von Ameln-Mayerhofer A, Berg D, Wagner HJ, et al. Effect of 6-hydroxydopamine (6-OHDA) on proliferation of glial cells in the rat cortex and striatum: evidence for de-differentiation of resident astrocytes. Cell and Tissue Research 2010; 342(2) 147-60.

[94] Bambrick L, Kristian T, Fiskum G. Astrocyte mitochondrial mechanisms of ischemic brain injury and neuroprotection. Neurochemical Research 2004; 29(3) 601-608.

[95] Dringen R. Metabolism and functions of glutathione in brain. Progress in Neurobiology 2000; 62(6) 649-671.

[96] Safi R, Gardaneh M, Panahi Y, Maghsoudi N, Zaefizadeh M, Gharib E. Optimized quantities of GDNF overexpressed by engineered astrocytes are critical for protection of neuroblastoma cells against 6-OHDA toxicity. The Journal of Molecular Neuroscience $2011 ; 46(3)$ 654-665.

[97] Zheng L, Ishii Y, Tokunaga A, Hamashima T, Shen J, Zhao QL, et al. Neuroprotective effects of PDGF against oxidative stress and the signaling pathway involved. The Journal of Neuroscience Research 2010; 88(6) 1273-1284.

[98] Ouyang YB, Xu LJ, Emery JF, Lee, AS, Giffard RG. Overexpressing GRP78 influences $\mathrm{Ca} 2+$ handling and function of mitochondria in astrocytes after ischemia-like stress. Mitochondrion 2011; 11(2) 279-286.

[99] Anderson CM, Swanson RA. Astrocyte glutamate transport: review of properties, regulation, and physiological functions. Glia 2000; 32(1) 1-14.

[100] Sims NR, Nilsson M, Muyderman H. Mitochondrial glutathione: a modulator of brain cell death. Journal of Bioenergetics and Biomembranes 2004; 36(4) 329-333.

[101] Lindenau J, Noack H, Possel H, Asayama K, Wolf G. Cellular distribution of superoxide dismutases in the rat CNS. Glia 2000; 29(1) 25-34.

[102] Hirrlinger J, Dringen R The cytosolic redox state of astrocytes: Maintenance, regulation and functional implications for metabolite trafficking. Brain Research Review 2010; 63(1-2) 177-188.

[103] Duncan AJ, Heales SJ. Nitric oxide and neurological disorders. Molecular Aspects of Medicine 2005; 26(1-2) 67-96.

[104] Giordano G, Kavanagh TJ, Costa LG. Mouse cerebellar astrocytes protect cerebellar granule neurons against toxicity of the polybrominated diphenyl ether (PBDE) mixture DE-71. Neurotoxicology 2009; 30(2) 326-329.

[105] Maier CM, Chan PH. Role of superoxide dismutases in oxidative damage and neurodegenerative disorders. Neuroscientist 2002; 8(4) 323-334.

[106] Slemmer JE, Shacka JJ, Sweeney MI, Weber JT. Antioxidants and free radical scavengers for the treatment of stroke, traumatic brain injury and aging. Current Medicinal Chemistry 2008; 15(4) 404-414. 
[107] Damier P, Hirsch EC, Zhang P, Agid Y, Javoy-Agid F. Glutathione peroxidase, glial cells and Parkinson's disease. Neuroscience 1993; 52(1) 1-6.

[108] Johnson JA, Johnson DA, Kraft AD, Calkins MJ, Jakel RJ, Vargas MR, et al. The Nrf2ARE pathway: an indicator and modulator of oxidative stress in neurodegeneration. Annals of the New York Academy of Sciences 2008; 1147 61-69.

[109] Martin HL, Teismann P. Glutathione-a review on its role and significance in Parkinson's disease. The FASEB Journal 2009; 23(10) 3263-3272

[110] Hauser RA, Lyons KE, McClain T, Carter S, Perlmutter D. Randomized, doubleblind, pilot evaluation of intravenous glutathione in Parkinson's disease. Movement Disorders 2009; 24(7) 979-83

[111] Chan PH. Role of oxidants in ischemic brain damage. Stroke 1996; 27(6) 1124-1129.

[112] Botella JA, Bayersdorfer F, Schneuwly S. Superoxide dismutase overexpression protects dopaminergic neurons in a Drosophila model of Parkinson's disease. Neurobiology of Disease 2008; 30(1) 65-73.

[113] Radunović A, Porto WG, Zeman S, Leigh PN. Increased mitochondrial superoxide dismutase activity in Parkinson's disease but not amyotrophic lateral sclerosis motor cortex. Neuroscience Letters 1997; 239(2-3) 105-108.

[114] Witt SN. Hsp70 molecular chaperones and Parkinson's disease. Biopolymers 2010; 93(3) 218-28.

[115] Kakizuka, A. Protein precipitation: a common etiology in neurodegenerative disorders? Trends in Genetics 1998; 14(10) 396-402.

[116] Giffard RG, Xu L, Zhao H, Carrico W, Ouyang Y, Qiao Y, et al. Chaperones, protein aggregation, and brain protection from hypoxic/ischemic injury. Journal of Experimental Biology 2004; 207(Pt 18) 3213-3220.

[117] Slodzinski H, Moran LB, Michael GJ, Wang B, Novoselov S, Cheetham ME, et al. Homocysteine-induced endoplasmic reticulum protein (herp) is up-regulated in parkinsonian substantia nigra and present in the core of Lewy bodies. Clinical Neuropathology 2009; 28(5) 333-43.

[118] Kalia SK, Kalia LV, McLean PJ. Molecular chaperones as rational drug targets for Parkinson's disease therapeutics. CNS \& Neurological Disorders-Drug Targets 2010; 9(6) 741-53.

[119] Bandopadhyay R, de Belleroche J. Pathogenesis of Parkinson's disease: emerging role of molecular chaperones. Trends in Molecular Medicine 2010; 16(1) 27-36.

[120] Kahle PJ, Waak J, Gasser T. DJ1 and prevention of oxidative stress in Parkinson's disease and other age related disorders. Free Radical Biology and Medicine 2009; 47(10) 1354-61. 
[121] Shendelman S, Jonason A, Martinat C, Leete T, Abeliovich A. DJ-1 is a redox-dependent molecular chaperone that inhibits alph $\alpha$-synuclein aggregate formation. PLoS Biol 2004; 2(11) e362.

[122] Klucken J, Shin Y, Masliah E, Hyman BT, McLean PJ. Hsp70 Reduces alph $\alpha$-synuclein aggregation and Toxicity. The Journal of Biological Chemistry 2004; 279(24) 25497-25502.

[123] Lee J, Giordano S, Zhang J. Autophagy, mitochondria and oxidative stress: cross-talk and redox signalling. The Biochemical Journal 2012; 441(2) 523-540.

[124] Mattson MP. Glutamate and neurotrophic factors in neuronal plasticity and disease. Annals of the New York Academy of Sciences 2008; 1144 97-112.

[125] Nutt JG, Burchiel KJ, Comella CL, Jankovic J, Lang AE, Laws ER, Jr., et al. Randomized, double-blind trial of glial cell line-derived neurotrophic factor (GDNF) in PD. Neurology 2003; 60(1) 69-73.

[126] Gill SS, Patel NK, Hotton GR, O'Sullivan K, McCarter R, Bunnage M, et al. Direct brain infusion of glial cell line-derived neurotrophic factor in Parkinson disease. Nature Medicine 2003; 9(5) 589-95.

[127] Patel NK, Bunnage M, Plaha P, Svendsen CN, Heywood P, Gill SS. Intraputamenal infusion of glial cell line-derived neurotrophic factor in PD: a two-year outcome study. Annals of Neurology 2005; 57(2) 298-302.

[128] Aberg ND, Brywe KG, Isgaard J. Aspects of growth hormone and insulin-like growth factor-I related to neuroprotection, regeneration, and functional plasticity in the adult brain. Scientific World Journal 2006; 6, 53-80.

[129] Pang, Y., Zheng, B., Campbell, L.R., Fan, L.W., Cai, Z., Rhodes, P.G. IGF-1 can either protect against or increase LPS-induced damage in the developing rat brain. Pediatric Research 2010; 67(6) 579-584.

[130] Kao SY. Rescue of alpha-synuclein cytotoxicity by insulin-like growth factors. Biochemical and Biophysical Research Communications 2009; 385(3) 434-438.

[131] Yasuhara T, Shingo T, Kobayashi K, Takeuchi A, Yano A, Muraoka K, et al. Neuroprotective effects of vascular endothelial growth factor (VEGF) upon dopaminergic neurons in a rat model of Parkinson's disease. European Journal of Neuroscience 2004; 19(6) 1494-1504.

[132] Yasuhara T, Shingo T, Muraoka K, wen Ji Y, Kameda M, Takeuchi A, et al. The differences between high and low-dose administration of VEGF to dopaminergic neurons of in vitro and in vivo Parkinson's disease model. Brain Research 2005; 1038(1) 1-10.

[133] Tian YY, Tang CJ, Wang JN, Feng Y, Chen XW, Wang L, et al. Favorable effects of VEGF gene transfer on a rat model of Parkinson disease using adeno-associated viral vectors. Neuroscience Letters 2007; 421(3) 239-244. 
[134] Timmer M, Muller-Ostermeyer F, Kloth V, Winkler C, Grothe C, Nikkhah G. Enhanced survival, reinervation, and functional recovery of intrastriatal dopamine grafts co-transplanted with Schwann cells overexpressing high molecular weight FGF-2 isoforms. Experimental Neurology 2004; 187(1) 118-136.

[135] Tang Z, Arjunan P, Lee C, Li Y, Kumar A, Hou X, et al. Survival effect of PDGF-CC rescues neurons from apoptosis in both brain and retina by regulating GSK3beta phosphorylation. Journal of Experimental Medicine 2010; 207(4) 867-880.

[136] Sullivan AM, Toulouse A. Neurotrophic factors for the treatment of Parkinson's disease. Cytokine \& Growth Factor Reviews 2011; 22(3) 157-65. 
